\title{
High-energy radiation and particles in the environments of young stellar objects
}

\author{
Manuel Güdel \\ Institute of Astronomy, ETH Zurich, 8093 Zurich, Switzerland \\ email: guedel@astro.phys.ethz.ch
}

\begin{abstract}
High-energy radiation and particles profoundly affect circumstellar disk gas and solids. We discuss stellar high-energy sources and summarize their effects on circumstellar disks.
\end{abstract}

Keywords. Young stellar objects, X-rays, high-energy particles, ionization

\section{Circumstellar disks subject to high-energy radiation and particles}

Stellar X-rays may heat circumstellar disks to $>1000 \mathrm{~K}$ at AU distances, as suggested by, e.g. $\mathrm{H}_{2} 2.12 \mu \mathrm{m}$ (Bary et al. 2003) and $\mathrm{CO}$ fundamental+overtone emission (Najita et al. 2003). X-rays may also ionize disks more efficiently than cosmic rays at similar distances, thus driving accretion through the magnetorotational instability (Glassgold et al. 1997). They may further dominate disk photoevaporation in the 10-40 AU range, resulting in mass loss rates of order $10^{-9} M_{\odot} \mathrm{yr}^{-1}$ (Ercolano et al. 2009).

What high-energy photons do reach the disk surface at all? Average neutral gas column densities around classical T Tauri stars (CTTS, $N_{\mathrm{H}} \approx 10^{21}-10^{22} \mathrm{~cm}^{-2}$, Güdel et al. 2007a) typically exceed those of weak-lined T Tauri stars, suggesting excess gas located relatively close to the star. This gas easily absorbs EUV radiation, as do disk winds (Hollenbach \& Gorti 2009), questioning the role of EUV radiation in CTTS disks. Absorption may be even more severe in some strong accretors where accretion streams seem to absorb all X-rays below $\approx 1-2 \mathrm{keV}$ (Güdel et al. 2007b).

Increasing X-ray absorption with increasing disk inclination provides, nevertheless, direct evidence for disk ionization (Kastner et al. 2005). Further support comes from fluorescent $6.4 \mathrm{keV} \mathrm{K} \alpha$ emission from "cold" iron at the disk surface during strong flares (Imanishi et al. 2001). Fluorescence may reach extremely high levels in protostars even outside any obvious flaring (Skinner et al. 2007).

The [Ne II] 12.81 $\mu \mathrm{m}$ line, frequently detected in Spitzer spectra (Pascucci et al. 2007), may be another diagnostic for X-ray/EUV disk irradiation (Glassgold et al. 2007). A statistically significant correlation with the stellar X-ray luminosity is, however, dominated by scatter (Figure 1a); obviously, a number of further parameters (e.g., disk properties) are relevant. For example, the presence of jets leads to a large increase in [Ne II] luminosity (Fig. 1b) as also shown in spatially resolved observations (van Boekel et al. 2009).

Glauser et al. (2009) found dust crystallinity to anti-correlate with the central star's $\mathrm{X}$-ray luminosity. Although X-rays carry insufficient momentum to induce lattice displacements, energetic (several keV) particles in the stellar wind are held responsible for amorphizing circumstellar dust.

\section{What high-energy sources?}

Apart from "traditional" magnetic coronae, additional high-energy sources have been identified. While accretion "suppresses" coronal X-rays by a factor of $\approx 2$ (Telleschi et al. 

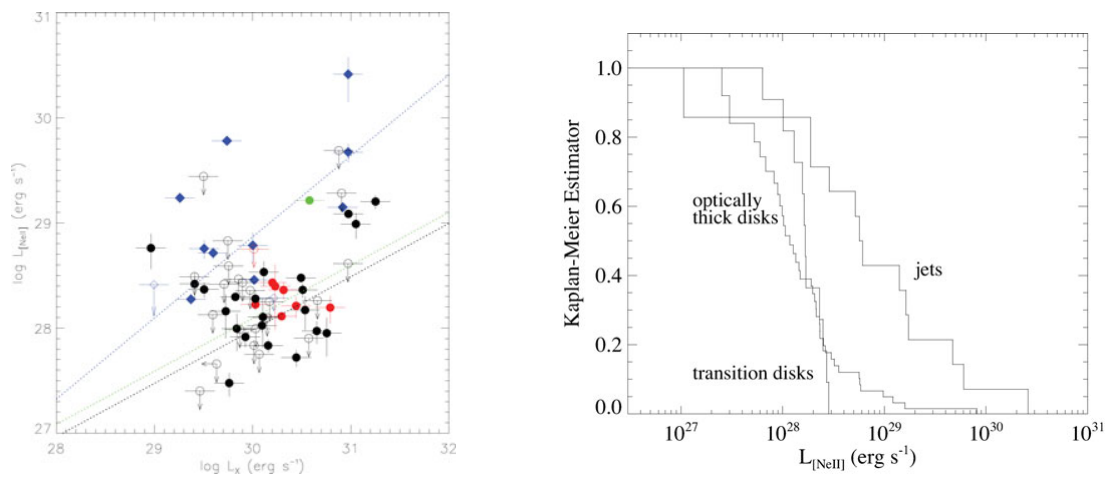

Figure 1. Left (a): [NeII] luminosity vs. X-ray luminosity for CTTS; jet sources are shown by diamonds, disks without jets by circles (filled: detections; open: upper limits). Right (b): Kaplan-Meier estimator for the [Ne II] luminosity of CTTS disks without jets, CTTS jet sources, and transition disks. From Güdel et al. (2009).

2007), it adds a "soft excess" at cool (coronal) temperatures (Güdel \& Telleschi 2007), perhaps due to an interaction between accretion streams and the corona. High densities inferred from X-ray line triplets (Kastner et al. 2002) have successfully been modeled in terms of shocks at the footpoints of accretion streams (Günther et al. 2007). CTTS jets also emit X-rays close to the star (Güdel et al. 2007b). Their "lamp-post" arrangement may provide ideal illumination of disk surfaces, avoiding absorption by accretion streams.

Large flares are of interest as they produce hard X-rays and may eject more energetic particles. Disks may thus be ionized more efficiently to deeper levels (Ilgner \& Nelson 2006). However, as the energetics of X-ray emission appear to be dominated by the large population of small flares (Audard et al. 2000), hard X-rays should be continuously present, adding as yet unrecognized ionization power to the circumstellar environment.

\section{References}

Audard, M., Güdel, M., Drake, J. J., \& Kashyap, V. L. 2000, ApJ 541, 396

Bary, J. S., Weintraub, D. A., \& Kastner, J. H. 2003, ApJ 586, 1136

Ercolano, B., Clarke, C. J., \& Drake, J. J. 2009, ApJ 699, 1639

Glassgold, A. E., Najita, J. R., \& Igea, J. 1997, ApJ 480, 344

Glassgold, A. E., Najita, J. R., \& Igea, J. 2007, ApJ 656, 515

Glauser, A. et al. 2009, A\&A, in press

Güdel, M. \& Telleschi, A. 2007, A\& $A$ 474, L25

Güdel, M. et al. 2007a, A\&̊A 468, 353

Güdel, M., et al. 2007b, A\&\&A 468, 515

Güdel et al. 2009, A\&\&A, submitted

Günther, H. M., Schmitt, J. H. M. M., Robrade, J., \& Liefke, C. 2007, A\&A, 466, 1111

Hollenbach, D. \& Gorti, U. 2009, ApJ, 703, 1203

Ilgner, M. \& Nelson, R. P. 2006, A\& A 455, 731

Imanishi, K., Koyama, K., \& Tsuboi, Y. 2001, ApJ 557, 747

Kastner, J. H. et al. 2002, ApJ 567, 434

Kastner, J. H. et al. 2005, ApJS 160, 511

Najita, J., Carr, J. S., \& Mathieu, R. D. 2003, ApJ, 589, 931

Pascucci, I. et al. 2007, ApJ, 663, 383

Skinner, S. L., Simmons, A. E., Audard, M., \& Güdel, M. 2007, ApJ 658, 1144

Telleschi, A., Güdel, M., Briggs, K. R., Audard, M., \& Palla, F. 2007, A\&\&A 468, 425

van Boekel, R., Güdel, M., Henning, Th., Lahuis, F., \& Pantin, E. 2009, A\&SA, 497, 137 